\title{
Comparative Study of Methods to Evaluate Depth of Cure of Restorative Composites
}

\author{
Beatriz C. Romano*, Jorge Soto, Beatriz C. Mendonça, Richard B. T. Price, Marcelo Giannini
}

\begin{abstract}
The objective of this study was to compare two methods to evaluate the depth of cure (DOC) of restorative composites and if the extension of the light-activation time would increase the DOC of composites. Two bulk-fill composites (Tetric Evoceram Bulk Fill, Ivoclar Vivadent and Filtek One Bulk Fill, 3M Oral Care) and two conventional composites (Tetric NCeram, Ivoclar Vivadent e Filtek Z350 XT, 3M Oral Care) were tested. Cylindrical samples were made by inserting the composites into a hole of matriz with $5 \mathrm{~mm}$ internal diameter and $13 \mathrm{~mm}$ depth, and light-activated with light curing unit from the same manufacturers of each composite, using the manufacturer recommendation time (MRT), or for double the time (DOT). Two methods compared were: 1- ISO 4049 test and 2- dissolution with organic solvent (acetone). For the ISO 4049 test, the unpolymerized resin was manually removed with a plastic spatula and the length of the samples measured with a digital caliper to calculate the DOC. In the organic solvent method, the unpolymerized resin was removed manually and samples were immersed inn the dark in acetone for 48 hours for DOC measurement. It was observed that light curing for DOT produced significantly higher DOC than those for MRT. Differences in DOC measurements between ISO and "organic solvent" methods were material dependent.
\end{abstract}

\section{Key words:}

Depth of cure, resin composites, light curing.

\section{Introduction}

Increased depth of cure (DOC) of bulk fill composites has been attributed to the development of new photoinitiators, new monomer compositions, particle modifications and the greater translucency of the material that allows deeper penetration of light from the light curing unit (LCU) into the restorative composite, allowing increments up to $4 \mathrm{~mm}$, without impairing the physical properties of the material. The ADA (American Dental Association) standardized a DOC test for composite resins, that measures the extent of polymerization in terms of cavity depth, using a scrapping method described by an international standard organization (ISO 4049). However, an alternative methods to evaluate the DOC of the resin-based composites (RBC) have been proposed, including chemical removal of poorly polymerized material with acetone, to replace scrapping and standardize the determination of polymerized material without external influence in the measurements.

\section{Results and Discussion}

Two conventional and two bulk fill materials were tested. The RBCs were inserted into $5 \mathrm{~mm}$ internal diameter, $13 \mathrm{~mm}$ depth plastic matrixes. The matrix was fixed over a polyester strip, on a glass plate lined with paper. Matrix was filled with a suprafil $1 / 2$ spatula and a polyester strip was placed over the surface of the composite. The light curing unit was fixed with the light guide tip parallel to the surface of the matrix and the RBCs were light-cured for the manufacturer's recommended time (MRT) or double the time (DOT).

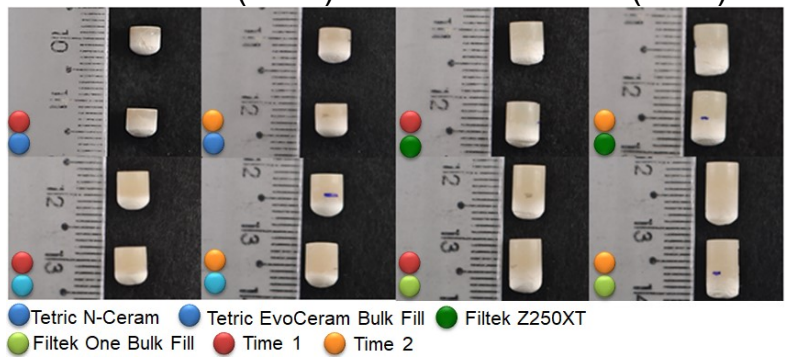

Image 1. Representative samples of each restorative material at each light curing time in the solvent removal method.
Table 1. Depth of cure of Tetric N-Ceram with different estimation methods and light curing times.

\begin{tabular}{|l|c|c|}
\hline & \multicolumn{2}{|c|}{ Method } \\
\hline Time & ISO & Solvent \\
\hline MRT & $2.26 \pm 0.08 \mathrm{Ab}$ & $1.19 \pm 0.05 \mathrm{Bb}$ \\
\hline DOT & $2.65 \pm 0.06 \mathrm{Aa}$ & $1.90 \pm 0.06 \mathrm{Ba}$ \\
\hline
\end{tabular}

Table 2. Depth of cure of Filtek Z350 XT with different estimation methods and light curing times.

\begin{tabular}{|l|c|c|}
\hline & \multicolumn{2}{|c|}{ Method } \\
\hline Time & ISO & Solvent \\
\hline MRT & $2.82 \pm 0.13 \mathrm{Ab}$ & $2.90 \pm 0.15 \mathrm{Ab}$ \\
\hline DOT & $3.23 \pm 0.06 \mathrm{Aa}$ & $3.69 \pm 0.13 \mathrm{Ba}$ \\
\hline
\end{tabular}

Table 3. Depth of cure of Tetric EvoCeram Bulk Fill with different estimation methods and light curing times.

\begin{tabular}{|l|c|c|}
\hline & \multicolumn{2}{|c|}{ Method } \\
\hline Time & ISO & Solvent \\
\hline MRT & $3.28 \pm 0.0 \mathrm{Ab}$ & $3.02 \pm 0.09 \mathrm{Bb}$ \\
\hline DOT & $3.83 \pm 0.08 \mathrm{Aa}$ & $3.81 \pm 0.11 \mathrm{Aa}$ \\
\hline
\end{tabular}

Chart 4. Depth of cure of Filtek One Bulk Fill depth of cure with different estimation methods and light curing times.

\begin{tabular}{|l|c|c|}
\hline & \multicolumn{2}{|c|}{ Method } \\
\hline Time & ISO & Solvent \\
\hline MRT & $4.00 \pm 0.18 \mathrm{Ab}$ & $4.02 \pm 0.28 \mathrm{Ab}$ \\
\hline DOT & $4.58 \pm 0.18 \mathrm{Ba}$ & $5.00 \pm 0.18 \mathrm{Aa}$ \\
\hline
\end{tabular}

For all tables, different letters indicate statistically significant differences. Upper case letters compare different methods within a same time. Lower case letters compare different times, within the same method.

\section{Conclusions}

A single method to determine DOC could not be standardized, as it depends on the materials composition, photoinitiators, and characteristics of the LCU.

\section{Acknowledgement}

\section{Support Funding Agency: FAPESP $N^{\circ}$ 2018/11976-2}

1. Rueggeberg FA. Determination of resin cure using infrared analysis without an internal standard. Dent Mater 10:282-286, July, 1994

2. Nomoto R., Uchida K. And Hirasawa T. Effect of Light Intensity on Polymerization of Light-cured Composite Resins. Dental Materials Journal 13(2): 198-205, 1994 